\title{
A influência do género e da ocasião na frequência de consumo de vinho
}

\author{
The role of gender and occasion on the frequency of wine consumption
}

\section{Carlos Peixeira Marques}

Universidade de Trás-os-Montes e Alto Douro, CETRAD, Quinta de Prados, 5000-801 Vila Real, Portugal, cmarques@utad.pt

\section{Ana Teresa Bernardo Guia}

Instituto Politécnico de Viseu, Escola Superior de Tecnologia e Gestão de Lamego, 5100-074 Lamego, Portugal, aguia@estgl.ipv.pt

\section{Resumo}

Este trabalho pretende avaliar de que modo a frequência de consumo de vinho depende do género do comprador e da ocasião a que se destina a compra. Para tal, foi desenvolvido e validado um modelo estrutural que incluiu o conhecimento subjetivo da categoria e as motivações para consumir como variáveis mediadoras. Os dados empíricos foram recolhidos de uma amostra de 523 compradores entrevistados após a compra em grandes superfícies retalhistas de Lisboa e Porto.

Os resultados indicam que a frequência de consumo é explicada predominantemente pela ocasião de consumo no contexto de refeição normal, havendo também um efeito muito significativo da motivação por gratificação sensorial. 0 conhecimento subjetivo revelou-se um elemento fundamental no comportamento do consumidor, quer pela sua influência sobre as motivações, quer pela sua influência sobre a frequência de consumo. Sugere-se que o marketing de vinhos para compradores regulares reforce a perceção de confiança e de eficácia de decisão que costumam ser associadas aos elevados níveis de conhecimento percebido numa categoria de produto.

Palavras-chave: comportamento do consumidor de vinho, conhecimento subjetivo, género, motivações para o consumo de vinhos, ocasião de consumo.

\section{Abstract}

To assess how the buyers' gender and the occasion in which the wine is consumed influence the frequency of consumption stated by the buyer, the research presented here has developed and validated a structural model that included as mediating variables the subjective knowledge regarding the category and motivations to consume. Empirical data was collected from a sample of 523 buyers just after purchasing in major retail areas of Lisbon and Oporto.

The results show that the frequency of consumption is explained mainly by consumption occasion in the context of an everyday meal, and that there is also a very significant effect of motivation for sensory gratification. The subjective knowledge proved to be a key element in consumer behaviour, both by its influence on the motivations and by its influence on the consumption frequency. It is suggested that wine marketing to regular buyers should reinforce their perception of selfefficacy, which is usually associated with high levels of perceived knowledge in a product category.

Key words: Wine consumer behavior, perceived knowledge, gender, motivation to consume wine, consumption occasions.

\section{Introdução}

O vinho é considerado um típico bem de experiência (Schamel \& Anderson, 2003) já que muitos dos seus atributos não são verificáveis antes do consumo, ou a procura de informação para os conhecer implica mais custos para o decisor do que a experiência direta com o produto (Nelson, 1970). Por outro lado, os mercados de vinhos são tendencialmente fragmentados (Berni, Begalli \& Capitello, 2005), com uma vasta gama de produtos associados a uma grande diversidade de marcas. Estes fatores contribuem para que o processo de escolha do consumidor se torne mais complexo; para que os atributos extrínsecos sejam mais importantes do que os intrínsecos; para que a perceção do consumidor sobre o seu conhecimento da categoria de produto tenha um papel mais importante do que a procura de informação (Park, Mothersbaugh \& Feick, 1994); e para que a ocasião de consumo assuma um papel fundamental no processo de decisão (Berni et al., 2005).

Para além da ocasião, o género do comprador é outro fator com influência significativa no processo de decisão de compra de bens de experiência (Park, Yoon \& Lee, 2009), considerando-se que, comparativamente às mulheres, os homens procuram fazer a aquisição com o mínimo de esforço na procura de informação (Laroche, Cleveland,
Bergeron \& Goutaland, 2003), apresentando-se como principal justificação o chamado modelo de seletividade as mulheres tendem a retirar gratificações do próprio processo de decisão em contexto de retalho, enquanto os homens tendem a encarar a compra como um problema e a obter de forma mais direta a solução (Campbell, 2000).

O principal objetivo do presente trabalho é apresentar e validar um modelo que considera os efeitos mediadores de variáveis que podem contribuir para explicar as influências do género e da ocasião na variação da frequência de consumo de vinho. 0 conhecimento subjetivo é um desses mediadores, tendo já sido estabelecido que, quer o género (Barber, Dodd \& Kolyesnikova, 2009), quer a ocasião (Barber, 2008), condicionam a importância do conhecimento no processo de decisão. Outro efeito mediador a considerar é o das motivações, também dependentes da ocasião (Hall, Lockshin, \& O'Mahony, 2001) e do género (Thach, 2012). Antes da formalização do modelo, proceder-se-á a uma revisão da literatura para enquadrar as variáveis consideradas, nomeadamente situando-as no contexto do processo de compra de vinhos.

\section{Revisão da literatura}

0 processo de escolha do vinho depara-se com alguma complexidade, situação que resulta, entre outros fatores, da 
alargada gama de produtos, da multiplicidade de marcas e da assimetria de informação existente neste tipo de mercado. Numa tentativa de ultrapassar este problema, o consumidor recorre às suas próprias construções de experiências vivenciadas, ao conhecimento (objetivo e subjetivo) e a diversas fontes de informação externas. No entanto, este recurso à informação é influenciado pela ocasião a que se destina a compra. Lai (1991) defende que o comportamento do consumidor é condicionado pela ocasião, a qual influencia o leque de marcas a analisar, o grau de profundidade da pesquisa, o tipo de informação recolhida e o orçamento para a compra. No caso específico do vinho, diferentes investigadores referem a ocasião como fator decisivo na escolha (Hall et al., 2001; Berni et al., 2005; Olsen, Thach \& Nowak, 2007).

A pesquisa bibliográfica efetuada denota a falta de consenso sobre uma tipologia de ocasiões de consumo. Por exemplo Dubow (1992) apresenta uma tipologia de base motivacional, distinguindo cinco tipos de ocasiões: duas introspetivas (para relaxar ou ajudar com o sono) e três sociais (para ser amigável, para ser aceite, ou para comemorar). Hall et al. (2001) utilizam cinco categorias de refeição: íntima; com amigos; em família; para comemorar; e relacionada com atividade profissional. Por seu turno, Thach (2011) refere: ocasiões formais, incluindo jantares formais e comemorações como aniversários, casamentos, etc.; e ocasiões informais, como refeições com amigos/ familiares ou simplesmente o consumo para socializar sem refeição.

Parece existir, também, uma relação entre género e a compra de vinho para determinadas ocasiões. Nicholson (1990) concluiu que as mulheres escolhem essencialmente vinho para refeições servidas em casa, enquanto os homens dominam na escolha de vinho para em ocasiões especiais. Barber et al. (2009) referem que as fontes de informação são percebidas de forma distinta pelos dois géneros, consoante a ocasião de consumo. Também Thach (2012) sugere uma interação entre género e ocasião na escolha do tipo de vinho a consumir. Para esta autora, a diferença de género no processo de escolha de vinho pode ser enquadrada num contexto motivacional, ou seja, homens e mulheres têm diferentes comportamentos no consumo de vinho porque têm motivações diferentes - os homens estão mais interessados em discutir os aspetos técnicos do vinho, exibindo o seu conhecimento, ao passo que as mulheres querem relaxar e socializar com os amigos.

A literatura sobre motivações para o consumo de vinho salienta a existência de motivos de várias ordens, como a gratificação sensorial, aprovação social, estimulação intelectual, entre outros. Hall et al. (2001) apresentam uma motivação negativa e um leque mais alargado de motivos positivos, como benefícios para a saúde, benefícios psicológicos, prazer, harmonização com a refeição, socialização e oportunidade de impressionar os outros. Na mesma linha, Charters e Pettigrew (2008) apresentam como principais motivos para consumir vinho o gosto, o gozo/prazer, a comida e a melhoria do humor. A estes acrescentaram a existência de alguns motivos simbólicos, como a auto-estima ou a projeção da própria imagem. Por sua vez, Thach (2012) invoca que, em situações de refeição, os principais motivos apontados pelos consumidores para beberem vinho são a melhoria da refeição e a apreciação do sabor do mesmo. No entanto, também são apontados motivos como o relaxamento, razões sociais, sofisticação e motivações de saúde.

No caso dos países mediterrânicos, a gratificação sensorial é referida como a principal motivação para o consumo de vinho (Barrena \& Sanchez, 2009). No entanto, os mesmos autores salientam o facto dos consumidores mais jovens serem motivados para o consumo de vinho por razões relacionadas com a identidade cultural e o status social. Num estudo aplicado ao caso específico do vinho em Portugal, Duarte, Madeira e Barreira (2010) definiram três dimensões de motivação, designadamente o gosto/prazer, a convivialidade e performance pessoal.

A literatura salienta o facto do conhecimento subjetivo nem sempre refletir o conhecimento objetivo (Dodd, Laverie, Wilcox \& Duhan, 2005 e Park et al., 1994). Conhecimento objetivo e subjetivo têm efeitos diferentes no comportamento do consumidor e na procura e processamento de informação. Park et al. (1994) sugeriram que a experiência com o produto está mais fortemente relacionada com o conhecimento subjetivo do que com o conhecimento objetivo. Brucks (1985) salienta que os consumidores com altos níveis de conhecimento subjetivo podem descartar alternativas que acreditam ser inferiores e sugere ainda que o conhecimento subjetivo se relaciona menos com o número de atributos examinados que o conhecimento objetivo, pois este encontra-se fortemente relacionado com a informação.

Algumas pesquisas identificaram diferenças entre homens e mulheres no que diz respeito ao processamento de informações e à tomada de decisão de compra. Laroche et al. (2003) sugerem que os homens são mais subjetivos e intuitivos nos processos de tomada de decisão. A experiência reforça a confiança dos homens no que diz respeito ao seu conhecimento subjetivo e este, por sua vez, facilita a tarefa de avaliação. No caso específico do vinho, Barber e Almanza (2006) evidenciam o facto dos homens se classificarem como mais informados sobre o vinho do que as mulheres, referindo ainda que as mulheres menos experientes estão mais preocupadas com a possibilidade de cometerem erros na escolha de vinho do que os homens. Estes resultados são congruentes com o modelo de seletividade, segundo a qual os homens tendem a seguir um processamento de informação mais heurístico do que as mulheres, o qual é bastante facilitado e reforçado por elevados níveis de conhecimento subjetivo.

\section{Modelo de análise e metodologia}

Este trabalho pretende avaliar em que medida o género e a ocasião de consumo influenciam a frequência de consumo de vinho. Na sequência da revisão da literatura, elabora-se o modelo de análise da Figura 1, com a finalidade de avaliar se o efeito destas variáveis exógenas é mediado pelas variáveis latentes conhecimento subjetivo e motivações. Ou seja, verificar-se-á empiricamente se a totalidade da variância comum entre a variável dependente (frequência de consumo) e as exógenas (género e ocasião) é devida aos mediadores (conhecimento e motivações) (Shrout \& Bolger, 2002). 


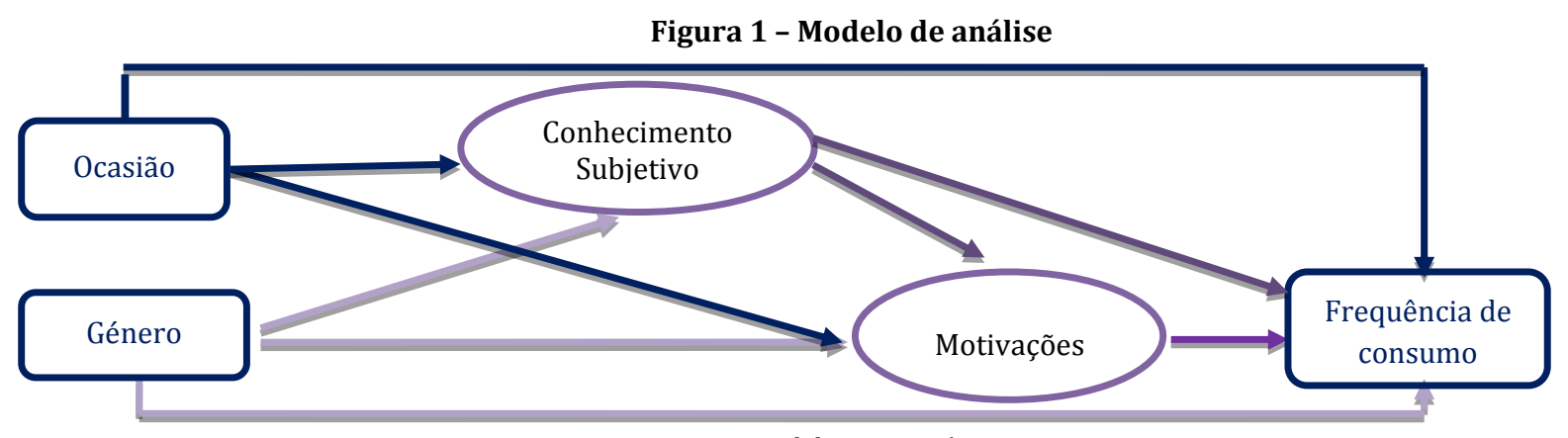

Fonte: Elaboração própria.

As relações entre as variáveis exógenas, os mediadores e a dependente, são apoiadas na literatura referida na secção anterior. Em alguns estudos desenvolvidos em Portugal (Duarte \& Ruivo, 1998; Teixeira, 2008) a frequência de consumo é referida como estando associada ao género, verificando-se que os homens consomem de forma mais regular do que as mulheres. A associação entre frequência de consumo e ocasião é implícita, pois se estivermos perante consumidores regulares (ou seja, que consomem diariamente ou mais do que uma vez por semana) é expetável que tal aconteça em refeições do dia-a-dia. Deduzse assim as seguintes hipóteses:

H1: Os homens consomem vinho mais frequentemente do que as mulheres.

H2: Os compradores de vinho para uma refeição normal consomem mais frequentemente do que os que compram para outras ocasiões.

A introdução do conhecimento subjetivo e das motivações como variáveis mediadoras na explicação da frequência de consumo introduz um caráter inovador a este modelo. As relações entre as variáveis exógenas e as mediadoras encontram-se fundamentadas na literatura. Propõe-se que o género masculino evidencie níveis de conhecimento subjetivo superiores ao feminino (Barber et al., 2009; Laroche et al., 2003 e Park et al., 2009). Quanto à influência da ocasião, considera-se que, comparativamente a outras ocasiões, o consumo numa refeição regular está associado a uma situação de compra de familiaridade, favorecendo-se assim a importância do conhecimento subjetivo (Barber, 2008 e Park et al. 1994). O conhecimento subjetivo é considerado um antecedente imediato de motivação (Park et al., 1994) e poderá mediar os efeitos do género (e.g. Thach, 2012) e da ocasião (e.g. Berni et al., 2005) sobre as motivações.

Quanto às relações entre os mediadores e a variável dependente, elas são, em parte, estabelecidas na literatura e, noutra parte, deduzidas a partir dela. Segundo Park et al. (1994) o conhecimento subjetivo está associado a maiores experiência e familiaridade com a categoria de produto, bem como a consumo mais intenso ou frequente. A gratificação sensorial, ao estar associada ao prazer de consumir vinho, ao gosto do sabor do vinho, à perceção de que o vinho ajuda a realçar do sabor dos alimentos numa refeição, induz uma maior frequência de consumo. Em sentido inverso, consumir por estimulação intelectual, ou seja, para tirar prazer do aumento do conhecimento da categoria, estará associado a uma menor frequência de consumo. 0 consumo de vinho por motivos sociais (aprovação social e conformação) está associado à partilha de momentos com amigos, de integração num grupo, o que implica um consumo menos frequente pois são, supostamente, situações que ocorrem de forma menos regular. Consequentemente, estabelece-se quatro hipóteses de relações entre os mediadores e a variável dependente:

H3: Os compradores com maior conhecimento subjetivo consomem mais frequentemente.

H4: A motivação por gratificação sensorial tem um efeito positivo sobre a frequência de consumo.

H5: A motivação por estimulação intelectual tem um efeito negativo sobre a frequência de consumo.

H6: A motivação social tem um efeito negativo sobre a frequência de consumo.

Para se proceder à verificação empírica do modelo de análise foi concebido um inquérito a uma amostra de 600 compradores de vinho numa cadeia de hipermercados nas regiões de Grande Lisboa e Grande Porto (300 inquiridos em cada região). 0 questionário, administrado por entrevistadores de uma empresa de estudos de mercado, obteve informações sobre o(s) vinho(s) adquirido(s); a (principal) ocasião a que se destinava(m); fatores que influenciaram a escolha; e fontes de informação utilizadas. Deste conjunto de questões, apenas a ocasião é considerada neste estudo. Na secção seguinte, o questionário incluiu as medidas das variáveis latentes representadas na Figura 1. Finalmente, uma terceira secção registou as caraterísticas sociodemográficas do comprador, entre as quais o género.

No que respeita à operacionalização das variáveis da Figura 1, a ocasião é uma variável dicotómica, medindo o efeito sobre as variáveis endógenas da ocasião "refeição normal" por comparação com as outras ocasiões. No caso do género, representa-se o efeito de ser homem, comparativamente a ser mulher. Quanto às variáveis latentes, os itens de conhecimento subjetivo seguem a proposta de Barber (2008), enquanto as motivações são representadas pelas três dimensões positivas propostas por Rossiter e Percy (1991): Intelectual (estímulos intelectuais, trocas de conhecimentos); Sensorial (despertar de sensações, prazer, gosto); Social (o consumo como facilitador da interação com o outro e da aprovação social). Partindo do trabalho de Henley e Donovan (2002), tendo também em consideração os itens utilizados por Duarte et al. (2010) e por Teixeira (2008), os autores desenvolveram 15 itens, cinco por cada dimensão, todos medidos numa escala de concordância de cinco pontos (1= concordo plenamente; $5=$ discordo plenamente). Os itens destas três dimensões, bem como o conhecimento subjetivo, foram avaliados num teste a 217 consumidores através de análise fatorial confirmatória, do qual resultou uma redução 
do tamanho das escalas utilizadas neste trabalho: as dimensões de motivação passaram de cinco a três itens cada, enquanto a variável conhecimento subjectivo passou de cinco a quatro. Para verificar se as variáveis exógenas exercem uma influência direta e/ou indireta sobre a frequência de consumo, os respetivos efeitos serão avaliados num modelo de equações estruturais com recurso a bootstraping, de acordo com as indicações de Shrout e Bolger (2002).

\section{Resultados}

A Tabela 1 sintetiza as principais caraterísticas dos 600 compradores inquiridos e das respetivas compras. Podemos verificar que os compradores têm uma idade média de 48 anos e são predominantemente homens (65\%), com curso superior (43\%), trabalhando por conta de outrem (51\%) e são consumidores regulares - 39\% fazem-no diariamente e $85 \%$ pelo menos uma vez por semana. Apesar desta frequência declarada, a quantidade adquirida no dia do inquérito foi reduzida, já que $2 / 3$ dos compradores adquiriram, no máximo, duas garrafas. A maioria comprou vinho tinto maduro (57\%) com destino a uma refeição normal (62\%).

Tabela 1 - Perfil do comprador de vinho

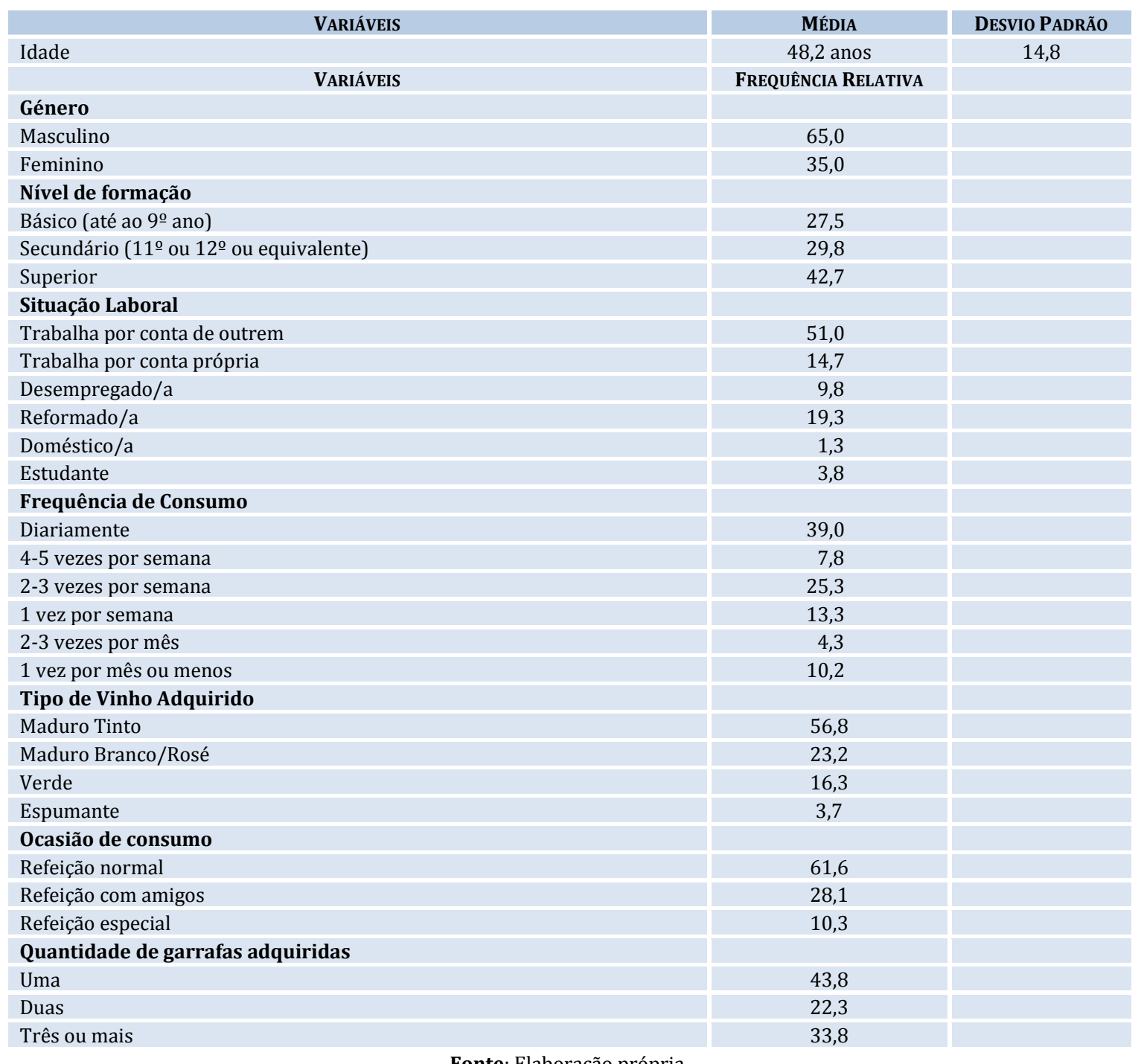

Fonte: Elaboração própria.

Antes de avaliar o modelo da Figura 1, é necessário validar as medidas das variáveis latentes. De acordo com as indicações habitualmente seguidas na literatura (Fornell \& Larcker, 1981; Hair, Black, Babin, \& Anderson, 2010), um constructo considera-se válido quando, cumulativamente: a escala é precisa (fiabilidade compósita maior do que 0,7); o constructo tem validez convergente (variância extraída média maior do que 0,5 ); há validez discriminante entre os diversos constructos ou dimensões (a média dos coeficientes padronizados de regressão de um fator com os respetivos itens é superior às correlações desse fator com qualquer um dos restantes).
Os três fatores de motivação, bem como o de conhecimento subjetivo, foram sujeitos a análise fatorial confirmatória com recurso ao software IBM SPSS Amos 21. Considerou-se apenas uma amostra de 523 compradores, correspondendo aos que responderam a todas as variáveis manifestas. 0 modelo de medida inicial revela uma qualidade de ajustamento que, de acordo com Marôco (2010) pode ser considerada sofrível: $\chi^{2} / g . l .=4,008$; CFI $=0,938 ; \mathrm{GFI}=0,936$; RMSEA=0,076; $\quad \mathrm{P}[\mathrm{RMSEA} \leq 0,05]<0,001$. Os índices de modificação sugeriam a retirada do modelo dos itens "Pretendo conhecer muitas marcas de vinho" e "O vinho facilita a minha interação com os outros " (pertencentes às 
dimensões Estimulação Intelectual e Social, respetivamente) por apresentarem relações cruzadas com outros fatores. Com a retirada destes itens do modelo, a sua bondade de ajustamento melhorou significativamente, apresentando agora uma boa qualidade: $\chi^{2} /$ g.l. $=2,911$; CFI $=0,969 ; \quad$ GFI $=0,964 ; \quad$ RMSEA $=0,061$; $\mathrm{P}[\mathrm{RMSEA} \leq 0,05]<0,091$. Como se pode verificar na Tabela 2 , todas as variáveis latentes apresentam $\mathrm{FC}>0,7$ e VEM $>0,5$.

Tabela 2 - Modelo de medida das variáveis latentes

\begin{tabular}{|c|c|c|c|c|c|c|}
\hline & & & MoDF & 13 ITENS & MoD & 11 ITENS \\
\hline ITENS (DIMENSÃO) & Média & DP & $\lambda(\mathbf{F C})$ & $\lambda^{2}($ VEM $)$ & $\lambda(\mathbf{F C})$ & $\lambda^{2}($ VEM $)$ \\
\hline GRATIFICAÇÃo SENSORIAL & & & 0,750 & 0,501 & 0,750 & 0,501 \\
\hline Gosto do sabor do vinho & 4,55 & 0,741 & 0,764 & 0,584 & 0,767 & 0,588 \\
\hline O vinho ajuda-me a apreciar melhor uma refeição. & 4,52 & 0,863 & 0,669 & 0,448 & 0,668 & 0,446 \\
\hline 0 vinho dá-me prazer. & 4,31 & 0,867 & 0,687 & 0,472 & 0,685 & 0,469 \\
\hline ESTIMULAÇÃO INTELECTUAL & & & 0,781 & 0,546 & 0,779 & 0,641 \\
\hline Quero saber mais sobre vinhos. & 3,74 & 1,013 & 0,741 & 0,549 & 0,715 & 0,511 \\
\hline $\begin{array}{l}\text { Gosto de aprender sobre as caraterísticas do vinho } \\
\text { associadas às várias castas. }\end{array}$ & 3,77 & 1,139 & 0,824 & 0,679 & 0,878 & 0,771 \\
\hline Pretendo conhecer muitas marcas de vinho. & 3,08 & 1,209 & 0,641 & 0,411 & & \\
\hline SOCIAL & & & 0,820 & 0,604 & 0,809 & 0,682 \\
\hline O vinho facilita a minha interação com os outros. & 3,01 & 1,284 & 0,701 & 0,491 & & \\
\hline $\begin{array}{l}\text { Beber vinho ajuda-me a sentir integrado no meu grupo } \\
\text { de amigos. }\end{array}$ & 2,50 & 1,273 & 0,815 & 0,664 & 0,722 & 0,521 \\
\hline O vinho ajuda-me a criar novas amizades & 2,30 & 1,273 & 0,810 & 0,656 & 0,918 & 0,843 \\
\hline CONHECIMENTo SubJETIVo & & & 0,838 & 0,569 & 0,838 & 0,569 \\
\hline $\begin{array}{l}\text { Conheço bem as caraterísticas dos vinhos das diversas } \\
\text { regiões portuguesas. }\end{array}$ & 3,58 & 1,095 & 0,656 & 0,430 & 0,659 & 0,434 \\
\hline Sei mais sobre vinho que a média das pessoas. & 2,84 & 1,138 & 0,855 & 0,731 & 0,855 & 0,731 \\
\hline $\begin{array}{l}\text { Sou muito conhecedor de assuntos relacionados com } \\
\text { vinho. }\end{array}$ & 2,85 & 1,152 & 0,840 & 0,706 & 0,839 & 0,704 \\
\hline Estou seguro de que sei escolher vinhos. & 3,45 & 1,115 & 0,639 & 0,408 & 0,638 & 0,407 \\
\hline
\end{tabular}

DP: desvio-padrão; $\lambda$ : peso fatorial; $\lambda^{2}$ : variância extraída; VEM: variância extraída média; FC: fiabilidade compósita; rótulos a negrito referem-se às linhas das dimensões; itens eliminados a itálico.

\section{Fonte: Elaboração própria}

Por sua vez, a Tabela 3 demonstra a validez discriminante de todas elas. Podemos, assim, considerar que estamos em presença de medidas válidas. É evidente nesta tabela que a gratificação sensorial é o fator de motivação mais saliente, sendo a motivação social pouco importante. Quanto ao conhecimento subjetivo, apresenta valores próximos do ponto médio da escala.

Tabela 3 - Correlações entre as variáveis latentes

\begin{tabular}{|c|c|c|c|c|}
\hline & $\begin{array}{c}\text { CONHECIMENTO } \\
\text { SUBJETIVO }\end{array}$ & $\begin{array}{c}\text { GRATIFICAÇÃo } \\
\text { SENSORIAL }\end{array}$ & $\begin{array}{c}\text { ESTIMULAÇ̃̃o } \\
\text { INTELECTUAL }\end{array}$ & SoCIAL \\
\hline CONHECIMENTO SUBJETIVO & $\mathbf{0 , 7 5 4}$ & & & \\
\hline GRATIFICAÇão SENSORIAL & 0,496 & $\mathbf{0 , 7 0 8}$ & & \\
\hline ESTIMULAÇão INTELECTUAL & 0,677 & 0,582 & $\mathbf{0 , 8 0 1}$ & \\
\hline SoCIAL & 0,290 & $-0,041$ & 0,165 & $\mathbf{0 , 8 2 6}$ \\
\hline
\end{tabular}

Os valores na diagonal são as médias dos pesos fatoriais Fonte: Elaboração própria.

Admitida a validade das medidas das variáveis latentes, o passo seguinte é a avaliação do modelo da Figura 1. Como foi referido, segue-se aqui as indicações de Shrout e Bolger (2002) para avaliar se as variáveis exógenas têm um efeito direto e/ou indireto sobre as variáveis dependentes, utilizando-se o método de bootstraping (com 2000 amostras) para calcular a significância e os intervalos de confiança dos estimadores dos efeitos diretos e indiretos. Incluindo todas as relações diretas possíveis na sequência variáveis exógenas $\rightarrow$ mediadores $\rightarrow$ variáveis dependentes, o modelo estrutural apresenta boa qualidade de ajustamento $\left(\chi^{2} /\right.$ g.l. $=3,017 ; \quad$ CFI=0,953; GFI=0,953; RMSEA=0,062; $\quad \mathrm{P}[\mathrm{RMSEA} \leq 0,05]<0,026)$. No entanto, constatou-se que nenhum dos efeitos diretos das variáveis exógenas era significativo $(0,182 \leq \mathrm{p} \leq 0,979)$, pelo que o modelo foi reconfigurado, considerando-se que o conhecimento subjetivo medeia completamente qualquer eventual efeito da ocasião ou do género sobre as motivações.

O novo modelo melhorou ligeiramente a sua bondade de ajustamento, como se pode comprovar pelas seguintes estatísticas e índices: $\chi^{2} / g . l=2,843 ; \mathrm{CFI}=0,953$; GFI=0,951; 
RMSEA=0,059; P[RMSEA $\leq 0,05]<0,058$. A Tabela 4 sintetiza os efeitos sobre a frequência de consumo e sobre as variáveis mediadoras, apresentando também os respetivos valores de variância explicada. Globalmente, o modelo explica cerca de $39 \%$ da variação da frequência de consumo. A ocasião apresenta-se como a variável com efeito direto mais significativo sobre a frequência, seguindo-se o efeito da gratificação sensorial. Com efeitos altamente significativos, mas de magnitude inferior, apresentam-se a estimulação intelectual (efeito negativo) e o conhecimento subjetivo (efeito positivo). Por último, verifica-se um efeito bastante reduzido do género, enquanto a motivação social não tem qualquer efeito.

Tabela 4 - Coeficientes estandardizados dos efeitos sobre as variáveis endógenas e respetivos $\mathrm{R}^{2}$ (estimativa bootstrap)

\begin{tabular}{|c|c|c|c|c|c|c|c|}
\hline & $\begin{array}{c}\text { REFEIÇÃo } \\
\text { NORMAL }\end{array}$ & НОМЕМ & $\begin{array}{c}\text { CONHECIMENTO } \\
\text { SUBJETIVO }\end{array}$ & SOCIAL & $\begin{array}{l}\text { GRATIFICAÇÃo } \\
\text { SENSORIAL }\end{array}$ & $\begin{array}{l}\text { ESTIMULAÇÃO } \\
\text { INTELECTUAL }\end{array}$ & $\mathbf{R}^{2}$ \\
\hline $\begin{array}{c}\text { CONHECIMENTO } \\
\text { SUBJETIVO }\end{array}$ & $0,214^{* *}$ & $0,252^{* *}$ & & & & & 0,112 \\
\hline SoCIAL & $0,065^{* *}$ & $0,077^{* *}$ & $0,305^{* *}$ & & & & 0,093 \\
\hline $\begin{array}{l}\text { GRATIFICAÇÃo } \\
\text { SENSORIAL }\end{array}$ & $0,106^{* *}$ & $0,125^{* *}$ & $0,497^{* *}$ & & & & 0,247 \\
\hline $\begin{array}{l}\text { ESTIMULAÇÃO } \\
\text { INTELECTUAL }\end{array}$ & $0,144^{* *}$ & $0,170^{* *}$ & $0,674^{* *}$ & & & & 0,454 \\
\hline \multirow{2}{*}{ FREQUÊNCIA } & $0,417^{* *}$ & $0,082^{*}$ & $0,239 * *$ & \multirow{2}{*}{$-0,040$} & \multirow{2}{*}{$0,380^{* *}$} & \multirow{2}{*}{$-0,262^{* *}$} & \multirow{2}{*}{0,391} \\
\hline & $0,051^{* *}$ & $0,060^{* *}$ & 0,000 & & & & \\
\hline
\end{tabular}

Recuperando as hipóteses estabelecidas, verifica-se que os resultados são compatíveis com todas, exceto H6, ou seja, apenas a motivação social não tem efeito direto sobre a frequência de consumo, todas as outras variáveis incluídas no modelo, quer as motivações, quer o conhecimento, quer as variáveis exógenas, influenciam diretamente a frequência. Tendo em conta os objetivos do presente trabalho, o resultado mais saliente é o fato das variáveis mediadoras não conseguirem explicar toda a variância comum entre as exógenas e a variável dependente, ou, dito de outro modo, o género do comprador e a ocasião a que se destina a compra, influenciam a frequência de consumo declarada pelo comprador, mesmo quando controlados (dum ponto de vista estatístico) os efeitos do conhecimento subjetivo e das motivações para consumir. Se no caso do género esta influência é pouco significativa, ela é bastante relevante quando o comprador declara ter adquirido vinho para uma refeição normal. Não obstante, os resultados merecem uma leitura mais complexa, no que respeita aos efeitos de mediação (Figura 2).

Figura 2 - Coeficientes estandardizados dos efeitos diretos

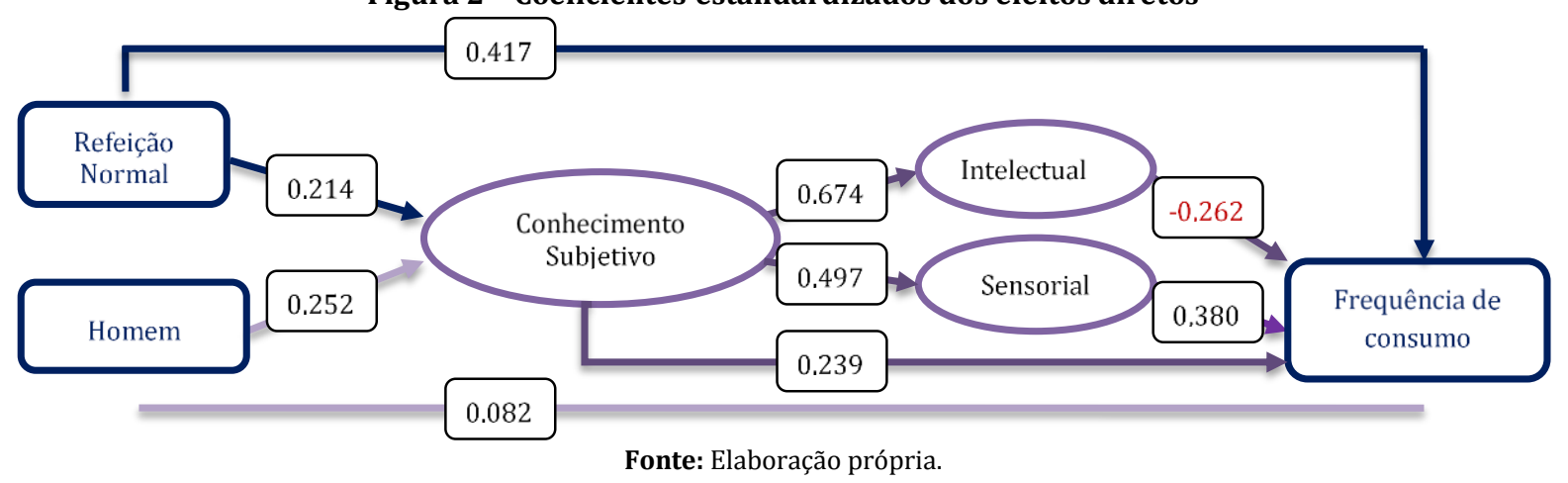

0 conhecimento subjetivo está associado à familiaridade com o vinho e é um facilitador na tomada de decisão de consumo (Laroche et al., 2003), não surpreendendo que exerça um efeito direto sobre a frequência de consumo. 0 conhecimento subjetivo favorece ainda a estimulação intelectual e a gratificação sensorial. Como estas motivações têm efeitos de sinal contrário sobre a frequência, há dois efeitos indiretos contraditórios do conhecimento sobre a frequência. 0 mesmo acontece com os efeitos indiretos do género e da ocasião, já que são antecedentes do conhecimento. Esta complexidade de relações pode resumir-se da seguinte forma: os homens e os compradores de vinho para refeições normais têm maior conhecimento de vinhos e estão mais motivados a consumir por estimulação intelectual, que tem efeito negativo na frequência, mas também por gratificação sensorial, que tem efeito positivo na frequência.

\section{Conclusões}

O principal objetivo do presente trabalho foi avaliar de que forma os efeitos mediadores do conhecimento subjetivo sobre vinhos e a motivação para consumir podem contribuir para explicar as influências do género e da ocasião na variação da frequência de consumo de vinho. Os resultados mais salientes indicam que: i) os compradores de vinho para uma refeição normal consomem mais frequentemente do que os que compram para outras ocasiões; ii) o conhecimento subjetivo e a gratificação 
sensorial exercem um efeito positivo sobre a frequência de consumo, enquanto a estimulação intelectual exerce um efeito negativo; iii) estes dois fatores de motivação contribuem de forma antagónica para os efeitos parciais de mediação da influência do género e da ocasião na frequência de consumo.

Este estudo apresenta vários contributos para a compreensão do consumo de vinhos. Em primeiro lugar, conseguiu-se operacionalizar com sucesso a dimensionalidade das motivações positivas para o consumo, de acordo com a teoria de Rossiter e Percy (1991). No entanto, os resultados mostram papéis completamente distintos destas três dimensões no modelo de análise que aqui foi avaliado. A gratificação sensorial é, na opinião dos inquiridos, a motivação que mais importância tem para os levar a consumir, na linha de estudos anteriores (Barrena \& Sanchez, 2009; Duarte et al., 2010), tendo também um efeito muito significativo na frequência de consumo. Pelo contrário, a motivação social, seja por interação, seja por integração, é considerada pouco importante e não tem qualquer efeito sobre a frequência, sendo também a menos explicada pelo conhecimento subjetivo. Quanto à estimulação intelectual, tem uma importância intermédia, tem um efeito moderado (negativo) sobre a frequência e tem uma relação forte com o conhecimento subjetivo.

Em segundo lugar, este estudo reforça o papel do conhecimento subjetivo no processo motivacional do consumo de vinho. Efetivamente, se os resultados forem extrapoláveis para o universo de compradores de vinho, poder-se-á dizer que as associações que têm sido referidas na literatura entre, por um lado, o género (Thach, 2012) e, por outro, a ocasião (Berni et al., 2005) e as motivações, são completamente mediadas pelo conhecimento subjetivo. Tal como esperado, o conhecimento é maior entre os compradores do género masculino (Barber, 2008; Barber \& Almanza, 2006; Laroche et al., 2003) e entre quem compra para refeições normais. Para além de exercer um efeito direto sobre a frequência de consumo, o conhecimento percebido tem um efeito determinante nas motivações para consumir, particularmente na estimulação intelectual, mas ainda muito significativo nas outras duas.

Em terceiro lugar, os resultados deste estudo mostram que o efeito direto do género sobre a frequência é bastante modesto, sugerindo que o maior conhecimento de vinhos declarado pelos homens contribua, em parte, para a maior frequência de consumo que tem sido referida na literatura (e.g. Duarte \& Ruivo, 1998).

Como é sabido, a experiência e familiaridade com a categoria de produto reforçam a confiança na decisão de compra, facilitando o processo de avaliação de alternativas (Laroche et al., 2003). Deste modo, os resultados aqui apresentados sugerem que o marketing de vinhos, nomeadamente os que se encontram à venda em hipermercados, reforce a confiança no conhecimento subjetivo do comprador, procurando associá-la à gratificação sensorial proporcionada pelo consumo. Este reforço das crenças no conhecimento e na eficácia da decisão, através da recompensa sensorial, parece-nos ainda mais pertinente se tivermos em conta que lidamos com um típico bem de experiência, uma categoria onde atributos extrínsecos tendem a ter um papel bastante relevante no processo de decisão.

Ao considerar apenas os compradores de vinho numa cadeia de hipermercados em Grande Lisboa e Grande Porto, a amostra denota uma sobre-representação do género masculino, do nível de formação superior e de consumidores diários de vinho. Há também uma maioria muito significativa de compradores que adquiriram vinhos para consumo no dia-a-dia, faltando um número suficiente de casos relativos a outras ocasiões de consumo para estimar de forma mais rigorosa os efeitos da ocasião, quer sobre a frequência de consumo, quer sobre as motivações. As limitações de amostragem, inerentes à escassez de recursos disponíveis para a realização deste trabalho, poderão ter alguma influência nos resultados, nomeadamente na irrelevância da motivação social. Sugerese que este tipo de estudo seja alargado a uma amostra nacional em diversos canais de distribuição, de modo a incluir segmentos que, eventualmente, dêem maior importância às dimensões social ou intelectual da motivação; tenham outro tipo de comportamento de compra, nomeadamente de maiores quantidades e preços mais elevados; e adquiram vinho para ocasiões associadas a maior envolvimento.

\section{Referências}

Barber, N., \& Almanza, B. (2006). Influence of wine packaging on consumers' decision to purchase. Journal of Foodservice Business Research, 9(4), 83-98.

Barber, N. (2008). How self-confidence and knowledge effects the sources of information selected during purchase situations. Ph.D. Thesis, Texas Tech University.

Barber, N., Dodd, T., \& Kolyesnikova, N. (2009). Gender differences in information search: Implications for retailing. Journal of Consumer Marketing, 26(6), 415-426.

Barrena, R., \& Sánchez, M. (2009). Using emotional benefits as a differentiation strategy in saturated markets. Psychology \& Marketing, 26(11), 1002-1030.

Berni, P., Begalli, D., \& Capitello, R. (2005). An occasion-based segmentation approach to the wine market in Denmark. Journal of International Food \& Agribusiness Marketing, 17(1), 117-145.

Brucks, M. (1985). The effects of product class knowledge on information search behavior. The Journal of Consumer Research, 12(1), 1-16

Campbell, C. (2000). Shopaholics, spendaholics, and the question of gender. In A. L. Benson (Ed.), I shop, therefore $i$ am: compulsive buying and the search for self (pp. 57-75). Northvale, NJ: Jason Aronson.

Charters, S., \& Pettigrew, S. (2008). Why do people drink wine? A consumer-focused exploration. Journal of Food Products Marketing, 14(3), 13-32.

Dodd, T., Laverie, D., Wilcox, J., \& Duhan, D. (2005). Differential effects of experience, subjective knowledge, and objective knowledge on sources of information used in consumer wine purchasing. Journal of Hospitality \& Tourism Research, 29(1), 3-19.

Duarte, F., Madeira, J., \& Barreira, M. (2010). Wine purchase and consumption in Portugal - an exploratory analysis of young adults' motives/attitudes and purchase attributes. Ciência e Técnica Vitivinicola, 25(2) 63-73.

Duarte, M., \& Ruivo, P. (1998). Buyer and consumer's Portuguese "Quality Wine" behaviour. Santarém: Instituto Superior de Agronomia e Instituto Politécnico de Santarém.

Dubow, J. (1992). Occasion based vs. user based segmentation. Journal of Advertising, 32(2), 11-18. 
Fornell, C., \& Larcker, D. F. (1981). Evaluating structural equation models with unobservable variables and measurement error. Journal of Marketing Research, 18(1), 39-50.

Hair, J., Black, W., Babin, B., \& Anderson, R. (2010). Multivariate data analysis $\left(7^{\text {th }}\right.$ ed.). Upper Saddle River, N.J.; Harlow: Pearson Education.

Hall, J., Lockshin, L., \& O'Mahony, G. B. (2001). Exploring the links between wine choice and dining occasions: Factors of influence. International Journal of Wine Marketing, 13(1), 36-53.

Henley, N., \& Donovan, R. (2002). Identifying appropriate motivations to encourage people to adopt healthy nutrition and physical activity behaviours. Journal of Research for Consumers, 4. Consultado junho 2015 em http://www.jrconsumers.com/academic_articles/issue_4/Henley Donovan.pdf

Lai, A. (1991). Consumption situation and product knowledge in the adoption of a new product. European Journal of Marketing, 25(10), 55-67.

Laroche, M., Cleveland, M., Bergeron, J., \& Goutaland, C. (2003). The knowledge experience-evaluation relationship: a structural equations modeling test of gender differences. Canadian Journal of Administrative Sciences, 20(3), 246-259.

Marôco, J. (2010). Análise de equações estruturais: fundamentos teóricos, software \& aplicações. Pêro Pinheiro: ReportNumber.

Nelson, P. (1970). Information and consumer behavior. Journal of Political Economy, 78(2), 311-329

Nicholson, P. (1990). Gender, power and wine selection: A pilot study. Journal of Wine Research, 1(3), 235-242.

Olsen, J., Thach, L., \& Nowak, L. (2007). Wine for my generation: exploring how US wine consumers are socialized to wine. Journal of Wine Research, 18(1), 1-18.

Park, C., Mothersbaugh, D., \& Feick, L. (1994). Consumer knowledge assessment. Journal of Consumer Research, 21(1), 71-82.

Park, J., Yoon, Y., \& Lee, B. (2009). The effect of gender and product categories on consumer online information search. Advances in consumer research, 36, 362-366

Rossiter, J., \& Percy, L. (1991, October 4-7, 1990). Emotions and motivations in advertising. Paper presented at the Advances in Consumer Research, 21, Provo, UT.

Schamel, G., \& Anderson, K. (2003). Wine quality and varietal, regional, and winery reputations: hedonic prices for Australia and New Zealand. Economic Record, 79(246), 357-369.

Shrout, P., \& Bolger, N. (2002). Mediation in experimental and nonexperimental studies: new procedures and recommendations. Psychological Methods, 7(4), 422-445.

Teixeira, C. (2008). O consumo de bebidas alcoólicas como lazer. Tese de Doutoramento, UTAD, Vila Real.

Thach, L. (2011). Wine for breakfast: exploring wine occasions for gen y. 6th AWBR International Conference. Bordeaux Management School.

Thach, L. (2012). Time for wine? Identifying differences in winedrinking occasions for male and female wine consumers. Journal of Wine Research, 23(2), 134-154. 